P06

ADVANCING CANCER PREVENTION PRICING INTERVENTIONS ACROSS THE UK AND US: OPTIMISING MESSAGE FRAMING - A QUALITATIVE FORMATIVE STUDY

${ }^{1} \mathrm{CH}$ Buckton*, ${ }^{1} \mathrm{~T}$ Ikegwuonu, ${ }^{2} \mathrm{JGL}$ Lee, ${ }^{3} \mathrm{R}$ Carey, ${ }^{4} \mathrm{JV}$ Cristello, ${ }^{4} \mathrm{EM}$ Trucco, ${ }^{5} \mathrm{~S}$ Golden, ${ }^{6} \mathrm{DI}$ Conway, ${ }^{1} \mathrm{~S}$ Hilton. ${ }^{1} \mathrm{MRC} / \mathrm{CSO}$ Social and Public Health Sciences Unit, University of Glasgow, Glasgow, UK; ${ }^{2}$ College of Health and Human Performance, East Carolina University, Greenville, USA; ${ }^{3}$ Health Psychology Research Group, University College London, London, UK; ${ }^{4}$ Department of Psychology, Florida International University, Miami, USA; ${ }^{5}$ Department of Health Behaviour, UNC Gillings School of Global Public Health, Chapel Hill, USA; ${ }^{6}$ School of Medicine Dentistry and Nursing, University of Glasgow, Glasgow, UK

\subsection{6/jech-2019-SSMabstracts. 158}

Background One-third of the burden of cancer is associated with four health harming behaviours - tobacco use, alcohol consumption, poor diet and physical inactivity. These individual behaviours are influenced by a complex array of sociopolitical factors, including industry interests. The growing evidence base supporting the use of price-related policies to curb over consumption of unhealthy products is of interest to governments around the world. However, the implementation of such policies requires public and policy-maker support. The aim of this study is to enhance our understanding of the features of message framing that influence public support in relation to four price-related policies (1-tax, 2-mitigation fee, 3-discounting ban and 4-minimum pricing); across three products (tobacco, alcohol and sugar); and two countries (USA and UK).

Methods We conducted 18 semi-structured interviews exploring knowledge, attitudes, and beliefs of the four pricing policies in relation to tobacco, alcohol and sugar products in 2018. Nine in the USA and nine in the UK. Interviewees included cancer policy advocates, cancer survivors and members of the general public. NVivo 12 was used to organise the data for inductive and deductive thematic analysis.

Results Common supportive features across all four policies included (i) highlighting the health benefit (including reduced cancer risk), particularly for young people and those on low income, arising from impact on purchasing behaviour and (ii) making the cost of consumption in terms of health harm clear. Common unsupportive features across policies included (i) that effectiveness will depend on size of the financial penalty and consumer response and (ii) such policies are unfair on sensible consumers and potentially regressive. Interviewees' were more supportive of price-related policies for tobacco containing products where they perceived a strong association between tobacco use and cancer risk. Some differences in views arose between products, particularly around the addictive nature of alcohol and tobacco that did not apply to sugar consumption.

Conclusion This study reveals important differences in levels of persuasiveness arising from the framing of messages designed to promote price-related policies as a health promotion strategy. The results have informed the identification of four frames to be tested in a multi-factorial quantitative study, currently under way. These frames can be characterised as: F1 - To reduce the financial strain on the health care system; F2 - For the protection of children and young people; F3 - To reduce cancer risk; and F4 - To reduce the risk of other non-communicable diseases.

\section{P08 \\ ARE CHANGES IN THE QUALITY AND QUANTITY OF SOCIAL SUPPORT ACROSS THE LIFE COURSE ASSOCIATED WITH CHANGES IN ALCOHOL CONSUMPTION? FINDINGS FROM THE WHITEHALL II STUDY}

P Case* ${ }^{*}$ A Britton. Epidemiology and Public Health, University College London, London, UK

\subsection{6/jech-2019-SSMabstracts.159}

Background Social support has been linked to positive health outcomes and one suggested mechanism for this effect is via health behaviours. Alcohol consumption is associated both with negative health and social outcomes and with positive social benefits, such as forming and maintaining friendships, relaxing and having fun.

A better understanding of which aspects of social support are associated with harmful alcohol consumption could help to improve public health messages and treatments for alcohol consumption; however, the existing evidence in this field is often contradictory and looks at the relationship at one time-point.

This study aimed to explore 1) the relationship between the quantity and quality of social support and harmful alcohol consumption, and 2) whether a change in the quantity and/or quality of social support over time is associated with a change in alcohol consumption.

Methods Data were from $6401 \mathrm{men} /$ women aged 35-55 at Phase 1 (1985) and 3704 men/women aged 47-67 at Phase 5 (1997) from a prospective cohort study of UK civil servants (Whitehall II). Measures of quantity and quality of social support from the Close Persons Questionnaire and last week alcohol consumption regrouped into non-drinkers; lower-risk $(\leq 14$ units/week); increasing-risk ( $>14$ to $\leq 35$ units/week-women, $>14$ to $\leq 50$ units/week-men) and higher-risk drinkers (>35 units/week-women, $>50$ units/week-men) were used to conduct multinomial logistic regression analyses.

Results

1) Amongst men, high levels of worsening support $(\mathrm{RRR}=1.95,95 \% \mathrm{CI}=1.13-3.37)$, seeing friends more often $(\mathrm{RRR}=2.20,95 \% \mathrm{CI}=1.10-4.42)$, seeing more friends/month and seeing relatives less frequently $(\mathrm{RRR}=1.89,95 \% \mathrm{CI}=1.12$ 3.19) increased the likelihood of being a higher-risk drinker.

Amongst women, seeing more friends/month $(R R R=4.21$, 95\% CI $=1.09-16.27)$ increased the likelihood, and being in the highest $(\mathrm{RRR}=0.25,95 \% \mathrm{CI}=0.06-0.95)$ and middle $(\mathrm{RRR}=0.21,95 \% \mathrm{CI}=0.06-0.79)$ tertiles for practical support reduced the likelihood of being a higher-risk drinker.

2) Amongst men, a reduction in confiding/emotional support $(\mathrm{RRR}=1.38,95 \% \mathrm{CI}=1.03-1.86)$ and an increase in practical support $(\mathrm{RRR}=1.54,95 \% \mathrm{CI}=1.01-2.37)$ were associated with a reduction in alcohol risk category. An increase in friends/ month $(\mathrm{RRR}=1.29,95 \% \mathrm{CI}=1.03-1.63)$ and a reduction in practical support $(\mathrm{RRR}=1.23,95 \% \mathrm{CI}=1.00-1.52)$ were associated with an increase in alcohol risk.

Amongst women, only a reduction in the number of close persons was associated with a reduction in alcohol risk group $(\mathrm{RRR}=1.74,95 \% \mathrm{CI}=1.05-2.87)$.

Conclusion Good levels of practical support appear particularly protective of harmful alcohol consumption, whereas seeing more of friends is associated with harmful alcohol use. Interventions which target friendship groups may be more effective 